\title{
Overview of the Proton-coupled MCT (SLC16A) Family of Transporters: Characterization, Function and Role in the Transport of the Drug of Abuse $\boldsymbol{\gamma}$-Hydroxybutyric Acid
}

\author{
Marilyn E. Morris ${ }^{1,2}$ and Melanie A. Felmlee ${ }^{1}$
}

Received 4 March 2008; accepted 1 April 2008; published online 4 June 2008

\begin{abstract}
The transport of monocarboxylates, such as lactate and pyruvate, is mediated by the SLC16A family of proton-linked membrane transport proteins known as monocarboxylate transporters (MCTs). Fourteen MCT-related genes have been identified in mammals and of these seven MCTs have been functionally characterized. Despite their sequence homology, only MCT1-4 have been demonstrated to be proton-dependent transporters of monocarboxylic acids. MCT6, MCT8 and MCT10 have been demonstrated to transport diuretics, thyroid hormones and aromatic amino acids, respectively. MCT1-4 vary in their regulation, tissue distribution and substrate/inhibitor specificity with MCT1 being the most extensively characterized isoform. Emerging evidence suggests that in addition to endogenous substrates, MCTs are involved in the transport of pharmaceutical agents, including $\gamma$-hydroxybuytrate (GHB), 3hydroxy-3-methyl-glutaryl-coenzyme A reductase inhibitors (statins), salicylic acid, and bumetanide. MCTs are expressed in a wide range of tissues including the liver, intestine, kidney and brain, and as such they have the potential to impact a number of processes contributing to the disposition of xenobiotic substrates. GHB has been extensively studied as a pharmaceutical substrate of MCTs; the renal clearance of GHB is dose-dependent with saturation of MCT-mediated reabsorption at high doses. Concomitant administration of GHB and L-lactate to rats results in an approximately two-fold increase in GHB renal clearance suggesting that inhibition of MCT1-mediated reabsorption of GHB may be an effective strategy for increasing renal and total GHB elimination in overdose situations. Further studies are required to more clearly define the role of MCTs on drug disposition and the potential for MCTmediated detoxification strategies in GHB overdose.
\end{abstract}

KEY WORDS: butyrate; gamma-hydroxybutyrate; lactate; monocarboxylate transporters; SLC16A.

\section{INTRODUCTION}

Monocarboxylic acids play a major physiological role in that they represent an energy source for all cells in the body. Of these compounds, lactate is critically important as it is the end product of glycolysis and intracellular accumulation of lactate results in the inhibition of glycolysis. Furthermore, lactate can be oxidized in the brain and red skeletal muscle to fuel cellular respiration. As such, the transport of lactate and other monocarboxylic acids both into and out of cells is vital for cellular function.

Two transporter families have been identified that facilitate this need: the proton-coupled monocarboxylate transporters (MCTs) and the sodium-coupled monocarboxylate transporters (SMCTs). MCTs (SLC16A) were first identified in the mid-nineties and to date 14 members of this

\footnotetext{
$\overline{{ }^{1} \text { Department of Pharmaceutical Sciences, School of Pharmacy and }}$ Pharmaceutical Sciences, University at Buffalo, State University of New York, Amherst, New York 14260, USA.

${ }^{2}$ To whom correspondence should be addressed. (e-mail: memorris @buffalo.edu)
}

family have been identified through sequence homology $(1,2)$. Currently, seven isoforms have been functionally characterized and it has been demonstrated that not all members function as proton-coupled transporters and that a wide variety of endogenous and exogenous compounds are substrates, including lactate, pyruvate, butyrate, $\gamma$-hydroxybutyrate, bumetanide, and simvastatin acid (3-6). In contrast, the SMCT family contains only two members, SLC5A8 and SLC5A12, which were identified within the past 5 years (7-9). SMCTs have strikingly similar substrate specificities transporting short-chain monocarboxylates and sodium ions with ratios between 4:1 and 2:1 (Na:substrate) (9). These two distinct transporter families are further differentiated by their respective tissue distributions: SMCTs demonstrate a more restricted distribution (primarily kidney and intestine) while MCTs show a more ubiquitous distribution $(4,9)$.

In addition, unlike SMCTs, some members of the MCT family have been demonstrated to transport exogenous compounds including drugs. The impact of MCT substrate/ inhibitor specificity and tissue distribution needs to be further examined with respect to drug substrates, and the overall influence of MCTs on drug disposition. The present review 
focuses on the proton-coupled MCTs and aims to summarize our current understanding of their structure, function and regulation as well as their role in drug disposition using $\gamma$ hydroxybutyrate (GHB; a known MCT substrate) (10-12) as a specific example.

\section{STRUCTURE, FUNCTION AND REGULATION OF MONOCARBOXYLATE TRANSPORTERS}

The uptake of monocarboxylates was first demonstrated to be transporter-mediated in erythrocytes $(13,14)$. Subsequently, the existence of a family of monocarboxylate transporters was proposed following the characterization of lactate transport in a variety of cell types $(13,15,16)$. To date, 14 members of the MCT family have been identified through screening of genomic and expressed sequence tag (EST) databases (4). Hydropathy plots have predicted that MCTs have 12 transmembrane domains with the $\mathrm{N}$ - and C-termini located in the cytoplasm $(2,4)$. The transmembrane domains (TMDs) are highly conserved between isoforms with the greatest sequence variations observed in the $\mathrm{C}$-terminus and the large intracellular loop between TMDs 6 and 7, which has a range of 29-105 amino acid residues (2). This observed variability is common to transporters with 12 TMDs and it is thought that these sequence variations are related to substrate specificity or regulation of transport activity $(2,17)$. Human tissue distribution of all currently identified isoforms has been investigated and is summarized in Table I. A number of recent reviews and articles have examined the tissue specific localization and physiological functions of MCT isoforms in both humans and rodents (18-25). Regulation of MCTs has been demonstrated to occur via transcriptional, translational and post-transcriptional mechanisms (26-28). These regulatory pathways appear to be age- and tissuedependent, which further complicates the understanding of these pathways $(27,28)$. Some MCTs require an ancillary protein (see Table I) which can be involved in cellular localization (29) or protein-protein interactions (30); however, the role of these accessory proteins in overall transporter function is not yet completely understood (29).

Functional characterization of MCT isoforms has been extended to seven isoforms (MCT1-4, 6, 8, 10) with the seven remaining MCT family members being classified as orphan MCTs (MCT5, 7, 9, 11-14). Table II provides a summary of currently identified substrates and inhibitors of functionally characterized MCT isoforms from humans and rats. Our current understanding indicates that the transport mechanism varies between MCT isoforms and that not all MCT isoforms transport monocarboxylates (e.g. MCT8). The following sections aim to provide an overview of our current understanding of individual MCT isoforms with respect to unique structural features, substrate/inhibitor specificity and regulation.

\section{MCT1}

MCT1 was first identified in Chinese hamster ovary cells when altered mevalonate transport resulting from a single point mutation was detected (15). Subsequently, human, rat and mouse homologues were cloned and functionally characterized (16,31-34). Tissue distribution of MCT1 is ubiquitous (Table I); however, localization within specific tissues varies.
For example, in the retinal pigment epithelium (RPE), expression is restricted to the apical membrane $(2,17)$. Transport kinetics have been thoroughly explored using lactate for this isoform and have demonstrated that it functions as a proton-dependent cotransporter/exchanger $(13,35)$. Transport occurs by ordered sequential binding with association of a proton followed by lactate binding. The complex is translocated across the membrane and the lactate and proton are released sequentially. Since the transporter functions as an exchanger, transport can occur bidirectionally; however, it is primarily responsible for the uptake of substrates (17).

While initial studies focused on the transport of lactate by MCT1, subsequent studies revealed that the substrate specificity of MCT1 was much less specific than initially thought $(2,4,35)$. Substrate and inhibitor affinities are detailed in Table II. Transport of lactate was shown to be stereoselective with MCT1 having a greater affinity for L-lactate than L-lactate (35). Uptake of butyrate by intestinal epithelia cells is highly dependent on MCT1 expression; alterations in MCT1 levels results in altered uptake of butyrate which is the primary energy source for these cells $(36,37)$. Interestingly, XP13512 (a gabapentin prodrug) was specifically designed to be a substrate for MCT1 in the intestine to improve the bioavailability of gabapentin $(38,39)$. In addition to the transport of short-chain monocarboxylic acids, MCT1 was demonstrated to transport branched oxo-acids with a greater affinity than lactate (35). The higher affinity of these acids for MCT1 supports previous studies demonstrating their inhibitory potential towards lactate transport. Inhibitors of MCT1 fall into three broad categories: (1) bulky or aromatic monocarboxylates which act as competitive inhibitors (e.g. phenyl-pyruvate and $\alpha$-cyano-4-hydroxycinnamate $(\mathrm{CHC})$ ); (2) amphiphilic compounds with divergent structures (e.g. quercetin and phloretin); and (3) some 4,4'-substituted stilbene-2,2'-disulphonates (e.g. DIDS) (4). Other isoforms can be distinguished from MCT1 based on the inhibitory potential of these compounds (Table II).

Relatively few studies have been conducted to assess the regulation of MCTs. Studies have indicated that altered physiological conditions and the presence of xenobiotics may alter the regulation of MCTs, in addition to altered expression at different developmental stages (40-42). MCT1 expression undergoes transcriptional, post-transcriptional and post-translational regulation and appears to be regulated in a tissue-specific manner (26-28). In colonic epithelium, exposure to butyrate resulted in a concentration- and timedependent increase in MCT1 mRNA, protein expression and a corresponding increase in butyrate transport (43). These data suggest the possibility of altered transcriptional regulation; however, the authors further demonstrated increased transcript stability indicating additional post-transcriptional regulation mechanisms (43). High concentrations of lactate have also been demonstrated to increase MCT1 mRNA and protein levels in L6 cells (44). In contrast, treatment with testosterone resulted in increased skeletal muscle MCT1 protein expression and lactate transport in the absence of mRNA changes suggesting the importance of post-transcriptional regulation (27). These results indicate that careful experimental design is required to assess the induction potential of exogenous compounds with respect to 


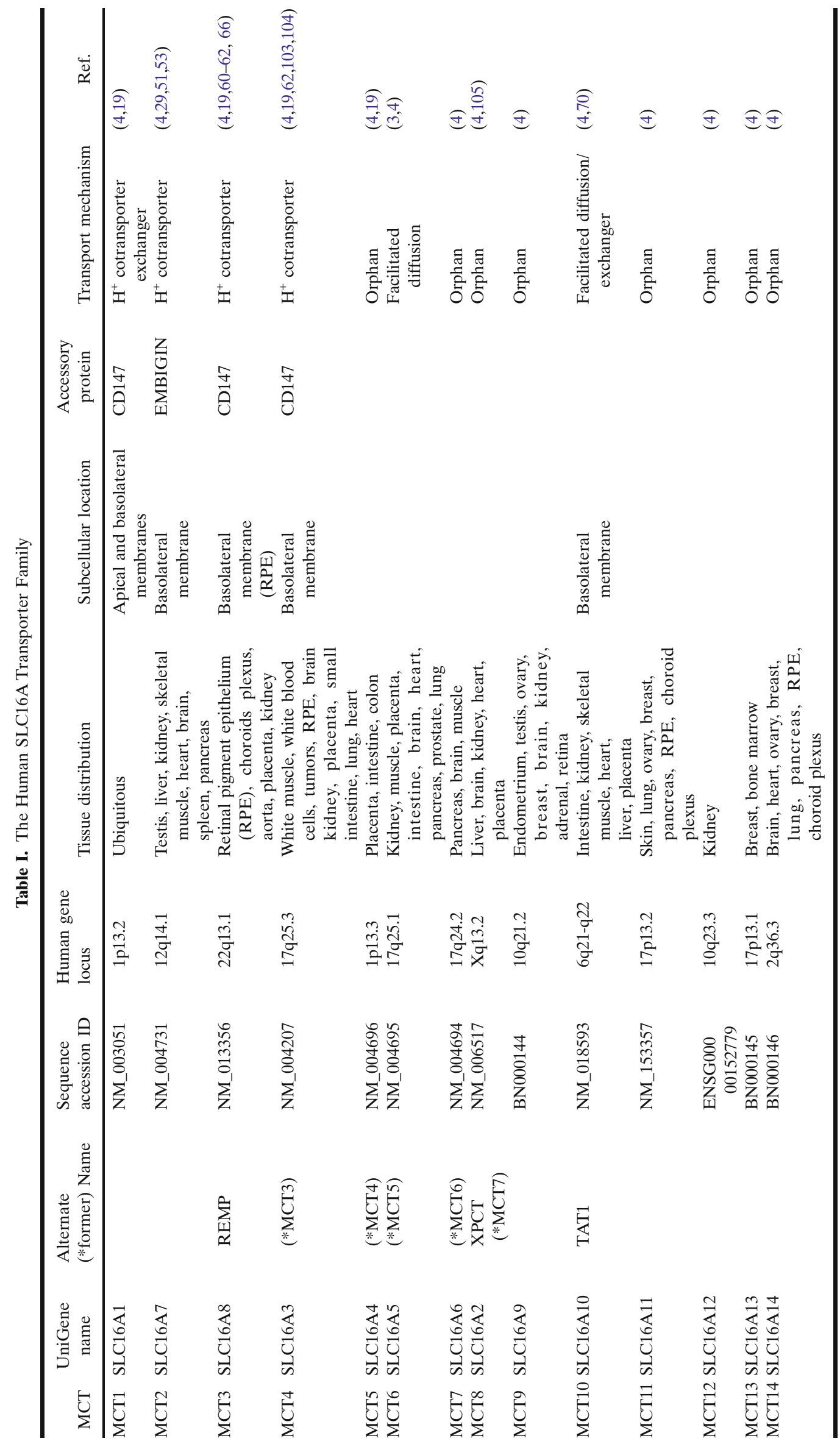


Table II. Comparison of Substrates and Inhibitors for Various MCT Isoforms in Humans and Rats

\begin{tabular}{|c|c|c|c|c|c|c|c|}
\hline Species & Isoform & Expression System & Substrate & $\mathrm{Km}(\mathrm{mM})$ & Inhibitor & $\begin{array}{l}\mathrm{Ki}^{\mathrm{a}} \text { or } \mathrm{IC} 50^{\mathrm{b}} \\
(\mu \mathrm{M})\end{array}$ & References \\
\hline \multirow[t]{5}{*}{$\overline{\text { Human }}$} & MCT1 & Xenopus oocytes & $\begin{array}{l}\text { Lactate } \\
\text { Pyruvate } \\
\text { Acetoacetate } \\
\alpha \text {-Ketoisovalerate } \\
\alpha \text {-oxoisohexanoate } \\
\alpha \text {-oxoisovalerate } \\
\text { Butyrate } \\
\text { XP13512 }\end{array}$ & $\begin{array}{l}3.5-6 \\
1.8-2.5 \\
5.5 \\
1.3 \\
0.67 \\
1.25 \\
9 \\
0.22\end{array}$ & $\begin{array}{l}\text { Phloretin } \\
\text { Quercetin } \\
\text { CHC } \\
\text { pCMBS } \\
\text { XP13512 }\end{array}$ & $\begin{array}{l}28^{\mathrm{a}} \\
\text { n.a. } \\
425^{\mathrm{a}} \\
\text { n.a. } \\
0.620^{\mathrm{b}}\end{array}$ & $(17,35,38,43,53)$ \\
\hline & $\mathrm{MCT} 2$ & Xenopus oocytes & Pyruvate & 0.025 & $\begin{array}{l}\mathrm{CHC} \\
\text { L-Lactate } \\
\text { GHB }\end{array}$ & $\begin{array}{l}\text { n.a. } \\
\text { n.a. } \\
\text { n.a. }\end{array}$ & (53) \\
\hline & $\begin{array}{l}\text { MCT3 } \\
\text { MCT4 }\end{array}$ & $\begin{array}{l}\text { ARPE-19 cells } \\
\text { Xenopus oocytes }\end{array}$ & $\begin{array}{l}\text { Lactate } \\
\text { L-lactate } \\
\text { D-lactate } \\
\text { Pyruvate } \\
\text { D- } \beta \text {-hydroxybutyrate } \\
\text { Acetoacetate } \\
\alpha \text {-ketobutyrate } \\
\alpha \text {-ketoisocaproate } \\
\alpha \text {-ketoisovalerate }\end{array}$ & $\begin{array}{l}\text { n.a. } \\
28 \\
519 \\
153 \\
130 \\
216 \\
57 \\
95 \\
113\end{array}$ & $\begin{array}{l}\text { pCMBS } \\
\text { CHC } \\
\text { Phloretin } \\
\text { NPPB } \\
\text { Fluvastatin } \\
\text { Atorvastatin } \\
\text { Lovastatin } \\
\text { Simvastatin }\end{array}$ & $\begin{array}{l}21^{\mathrm{a}} \\
991^{\mathrm{a}} \\
41^{\mathrm{a}} \\
240^{\mathrm{a}} \\
32^{\mathrm{b}} \\
32^{\mathrm{b}} \\
44^{\mathrm{b}} \\
79^{\mathrm{b}}\end{array}$ & $\begin{array}{l}(21) \\
(64,65)\end{array}$ \\
\hline & MCT6 & Xenopus oocytes & $\begin{array}{l}\text { Bumetanide } \\
\text { Nateglinide } \\
\text { Prostaglandin } F_{2} \alpha\end{array}$ & $\begin{array}{l}0.084 \\
\text { n.a. } \\
\text { n.a. }\end{array}$ & $\begin{array}{l}\text { Furosemide } \\
\text { Azosemide }\end{array}$ & $\begin{array}{l}46^{\mathrm{b}} \\
21^{\mathrm{b}}\end{array}$ & (3) \\
\hline & МCT8 & COS1 and JEG3 cells & $\begin{array}{l}\mathrm{T}_{3} \\
\mathrm{~T}_{4}\end{array}$ & $\begin{array}{l}\text { n.a. } \\
\text { n.a. }\end{array}$ & & & $(72)$ \\
\hline \multirow[t]{4}{*}{ Rat } & MCT1 & Xenopus oocytes & $\begin{array}{l}\text { Lactate } \\
\text { GHB }\end{array}$ & $\begin{array}{l}3.5 \\
4.6\end{array}$ & $\begin{array}{l}\text { Phloretin } \\
\text { Quercetin } \\
\text { Benzbromaron } \\
\text { CHC }\end{array}$ & $\begin{array}{l}28^{\mathrm{b}} \\
14^{\mathrm{b}} \\
22^{\mathrm{b}} \\
425^{\mathrm{b}}\end{array}$ & $(12,35,58)$ \\
\hline & MCT2 & $\begin{array}{l}\text { MDA-MB231 } \\
\text { Xenopus oocytes }\end{array}$ & $\begin{array}{l}\gamma \text {-hydroxybutyrate } \\
\text { Lactate } \\
\text { Pyruvate }\end{array}$ & $\begin{array}{l}4.6 \\
0.74 \\
\text { n.a. }\end{array}$ & $\begin{array}{l}\text { Phloretin } \\
\text { Quercetin } \\
\text { Benzbromaron } \\
\text { CHC }\end{array}$ & $\begin{array}{l}14^{\mathrm{b}} \\
5^{\mathrm{b}} \\
9^{\mathrm{b}} \\
24^{\mathrm{b}}\end{array}$ & $\begin{array}{l}(12) \\
(58)\end{array}$ \\
\hline & MCT4 & Xenopus oocytes & $\begin{array}{l}\text { L-lactate } \\
\text { Pyruvate } \\
\text { 2-oxoisohexanoate } \\
\text { Acetoacetate } \\
\beta \text {-hydroxybutyrate }\end{array}$ & $\begin{array}{l}34 \\
36.3 \\
13 \\
31 \\
65\end{array}$ & $\begin{array}{l}\text { CHC } \\
\text { pCMBS }\end{array}$ & $\begin{array}{l}350^{\mathrm{b}} \\
\text { n.a. }\end{array}$ & (104) \\
\hline & MCT10 & Xenopus oocytes & $\begin{array}{l}\mathrm{T}_{3} \\
\mathrm{~T}_{4} \\
\text { L-Tryptophan } \\
\text { L-Tyrosine } \\
\text { L-Phenylalanine } \\
\text { L-DOPA }\end{array}$ & $\begin{array}{l}\text { n.a. } \\
\text { n.a. } \\
3.8 \\
2.6 \\
7.0 \\
6.4\end{array}$ & $\begin{array}{l}N \text {-bromoacetyl- } \mathrm{T}_{3} \\
\text { Bromosulfophthalein }\end{array}$ & $\begin{array}{l}\text { n.a. } \\
\text { n.a. }\end{array}$ & (71) \\
\hline
\end{tabular}

CHC $\alpha$-Cyano-4-hydroxycinnamate, NPPB 5-nitro-2-(3-phenylpropylamino)benzoate, $p C M B S$ p-chloromercuribenzenesulphonic acid, n.a. transporter kinetic parameters were not determined

The superscripts are used with the data in the same column of the table to indicate if the values are IC50 or Ki values

MCT1 and multiple regulation pathways appear to be involved in its regulation. The MCT1 5'-flanking and 3' untranslated regions were recently cloned and a variety of transcription factor binding sites were identified (26). In addition, increased MCT1 expression and activity have been reported in human neuroblastoma and melanoma cell lines resulting from low extracellular $\mathrm{pH}(41,45)$. Inhibition and silencing of MCT1 in neuroblastoma and glioma cell lines resulted in increased cellular $\mathrm{pH}$ leading to apoptotic cell death suggesting that MCT1 may represent a novel chemo- therapeutic target $(41,46,47)$. Additional studies need to address the potential for varied physiological states and xenobiotics to alter MCT1 (or other isoforms) regulation, as this may impact the disposition of both endogenous and exogenous MCT substrates.

MCT1 is further regulated by its association with the cell surface glycoprotein CD147, which has a single transmembrane domain with the C-terminus located in the cytosol $(48,49)$. Topology studies suggest that one MCT1 molecule interacts with a single CD147 molecule with subsequent 
dimerization with another MCT1/CD147 pair.(49). The initial association of CD147 and MCT1 is required for the translocation of MCT1 to the plasma membrane (48). Furthermore, studies indicate that covalent modification of CD147 results in inhibition of lactate transport as is seen with pCMBSmediated inhibition of transport $(48,50)$. In addition to MCT1, CD147 functions as an ancillary protein for MCT4 but not MCT2 (48).

\section{MCT2}

MCT2 was initially isolated and functionally characterized from a Syrian hamster liver library (51) with subsequent identification of homologues in rat (52) and human (53). In humans, expression of MCT2 is more restricted than MCT1 (Table I), with the greatest expression observed in the testis (53). In addition, species differences have been observed in the tissue distribution of MCT2. For example, rodents express higher levels of MCT2 in the liver, while MCT2 protein expression is not detectable in human liver (53). Brain MCT2 expression and cellular localization also appears to be highly species dependent (53-55). This variability in tissue expression may be a result of species differences in gene regulation. In both rodents and humans, MCT2 splice variants have been detected in a species and tissue-dependent manner suggesting that transcriptional and post-transcriptional regulation pathways play an important role in the tissue specificity of this isoform $(52,53,55,56)$. Similar to MCT1, MCT2 requires an accessory protein for translocation to the plasma membrane. However, MCT2 requires gp70 (EMBIGIN), not CD147 (29). In addition, tissue specific post-translational regulation of MCT2 has recently been demonstrated in the mouse brain with the association of MCT2 and the scaffolding protein Delphilin which results in colocalization of MCT2 with $\delta$ glutamate receptors $(30,57)$. Further studies on the speciesand tissue-specific regulation are required to identify the complex pathways involved in MCT2 regulation.

MCT2 has remarkably similar substrate specificity to MCT1. However, in contrast to the observed substrate affinities of MCT1, MCT2 was demonstrated to be a high affinity pyruvate transporter in humans $\left(K_{\mathrm{m}}=25 \mu \mathrm{M}\right)$ which concurs with results obtained using hamster and rat MCT2 (Table II) $(51,58)$. Furthermore, MCT2 is inhibited by phloretin and $\mathrm{CHC}$, but not by the organomercurial thiol reagent pCMBS, which distinguishes it from MCT1 (4). It is thought that this difference in inhibitor sensitivity results from the requirement of MCT1 and MCT2 for different accessory proteins (4).

\section{MCT3}

MCT3 is believed to have the most restricted distribution of any MCT with expression in the basolateral membrane of the RPE and the choroid plexus in humans, rodents and chickens $(21,59,60)$. However, recent studies demonstrated MCT3 expression in vascular smooth muscle cell lines (61), human aorta (61), human kidney (62) and human intestinal Caco-2 cells (unpublished), suggesting that MCT3 mRNA may be more widely distributed than originally thought. Furthermore, decreased MCT3 mRNA and protein expression was observed with increasing severity of atherosclerosis which concurs with changes in smooth muscle cells characteristic of this disease state (61). The authors further demonstrated that DNA methylation of the MCT3 gene likely contributed to the observed expression changes (61).

Chicken MCT3 has been demonstrated to transport lactate in a yeast expression system $\left(K_{\mathrm{m}}=6 \mathrm{mM}\right)$ and demonstrates a profound resistance to prototypical MCT inhibitors (60). Additional information on human MCT3 substrates or inhibitors is not present in the literature nor is there detailed information regarding the regulation of MCT3.

\section{MCT4}

MCT4 demonstrates remarkable similarities to MCT1 with respect to tissue distribution, regulation and substrate/ inhibitor specificity (Tables I and II). The principal difference between these isoforms lies in their tissue specific localization and substrate affinities. In contrast to MCT1, MCT4 is predominantly expressed in highly glycolytic cells such as white muscle and white blood cells suggesting that its physiological function is lactate efflux $(17,63)$. MCT4 and CD147 expression were induced in MDA-MB231 cells (a highly invasive breast cancer cell line) supporting the metabolic switch to highly glycolytic cells in metastasis and the corresponding increase in lactate efflux (42). MCT4 localization at the plasma membrane was dependent on CD147 expression, which is consistent with results obtained for MCT1 (42). The role of MCT4 in lactate efflux is further supported by its high expression in the placenta where it is involved in the transfer of lactate into the maternal circulation (4). While there is a great degree of overlap in the substrate specificity of MCT1 and MCT4, these two isoforms differ in their substrate affinities with MCT4 having lower affinities for a range of monocarboxylates (64). In contrast to other MCTs, lactate transport via MCT4 is inhibited by a range of statin drugs which may play a role in cytotoxicities observed with statin administration (65).

\section{MCT6}

MCT6 was first identified by Price et al. in 1998 (66) through genomic and EST database screening. Northern blot analysis was used to determine the tissue distribution of MCT6 (Table I) with expression being predominantly in the kidneys (66).

In contrast to other members of the MCT family, MCT6 does not transport short-chain monocarboxylates or amino acids; rather, all substrates identified to date are pharmaceutical agents (Table II) (3). Murakami et al. (3) demonstrated that bumetanide uptake is mediated by MCT6 in a pH- and membrane potential-, but not proton-dependent manner suggesting that it may be net charge dependent. Furthermore, uptake of bumetanide was inhibited by probenecid and several thiazides, but not inhibited by lactate or succinic acid (3). This suggests that a carboxylic moiety is not essential for MCT6 affinity, as was anticipated based on results obtained with other MCT isoforms (3). MCT6 mRNA expression has been demonstrated along the entire length of the human intestine with the highest expression levels observed in the stomach $(66,67)$. This expression pattern suggests that MCT6 may play an important role in the intestinal absorption of 
xenobiotics. Further studies are required to determine the physiological role of MCT6 as well as its role in drug disposition.

\section{MCT8 and MCT10}

MCT8 was identified during studies on X-chromosome inactivation, and was previously known as X-linked PESTcontaining transporter due to the presence of a PEST domain in the N-terminus of the protein $(2,68)$. The gene encoding human MCT8 (hSLC16A2) contains two translation start sites either of which would result in a functional protein; it is currently unknown if these sites encode different MCT8 isoforms, and whether this would alter the isoforms function or regulation (69). Interestingly, in other species studied, SLC16A2 contains only a single start site that corresponds to the second site in the human gene (69). Further genomic analyses revealed a remarkable homology (52\% amino acid sequence identity) (69) between MCT8 and the T-type amino acid transporter-1, now known as MCT10. MCT10 contains a PEST domain within its N-terminus, a structural feature that is present in only MCT8 and MCT10, which is thought to result in rapid protein degradation (69).

Both MCT8 and MCT10 demonstrate a wide tissue distribution (Table I). The recent functional characterization of MCT8 and MCT10 revealed that monocarboxylates, including lactate and pyruvate, were not substrates for these transporters (69-71). MCT8 was demonstrated to actively transport the thyroid hormones, $\mathrm{T}_{3}$ and $\mathrm{T}_{4}(71,72)$, while MCT10 is involved in the transport of aromatic amines (70). The substrate specificity of MCT8 has further been confirmed by a linkage between mutations in MCT8 and AllanHerndon-Dudley Syndrome which is associated with abnormally high levels of circulating $\mathrm{T}_{3}$ (73). Both isoforms have been demonstrated to transport their respective substrates in a proton- and $\mathrm{Na}^{+}$-independent manner (70), which is in contrast to other members of MCT family. Interestingly, MCT10mediated transport of aromatic amines in the kidney has been demonstrated to occur in both directions thereby equalizing intra- and extracellular amino acid concentrations (69).

\section{Orphan MCTs}

Seven additional members of the MCT family (MCT5, MCT7, MCT9, AND MCT11-14) have been identified through searches of the human genomic and EST databases $(4,66)$. Table I details the human tissue distribution of these MCT isoforms as determined by Northern blot analyses $(4,66)$; limited data is available on the tissue-dependent protein expression of these isoforms (17). MCT5 protein expression has been demonstrated in the basolateral membrane of human colon and ileum with the greatest expression observed in the distal colon (19). It remains unclear whether monocarboxylates are substrates for these transporters. Riboflavin has been suggested as a substrate for MCT12 based on its sequence homology to Mch5p, which is responsible for plasma membrane uptake of riboflavin in Saccharomyces cerevisiae (74). However, functional characterizations of the orphan MCTs have yet to be completed. Until recently, no information was available regarding the regula- tion of the orphan MCTs. Hirai et al. (75) demonstrated that MCT13 was induced by PPAR- $\alpha$ agonists in mouse liver and small intestine suggesting that this transporter may be involved in nutrient uptake. Further studies are required to elucidate the exact mechanism of induction via this pathway and the role of PPAR- $\alpha$ in the overall regulation of MCT13.

\section{ROLE OF MCTS IN DRUG DISPOSITION}

Studies examining MCTs have focused primarily on their identification and understanding their physiological role in lactate homeostasis as well as the transport of additional endogenous substances; however, emerging evidence supports the further investigation of the impact of MCTs on drug disposition. For example, functional characterization of MCT6 indicated that it was involved in the transport of bumetanide, and not endogenous monocarboxylates (3). Furthermore, GHB has been demonstrated to be both a substrate and inhibitor for a number of MCT isoforms (10-12,53).

MCTs are expressed in a wide range of tissues, including the liver, kidney, intestine and brain (4). This localization has the potential to impact a number of processes contributing to the overall pharmacokinetics and distribution of therapeutic agents. Specifically, inhibition of renal reabsorption via MCTs results in increased renal clearance and decreased drug exposure. In addition, inhibition of MCT-mediated intestinal absorption may substantially decrease drug bioavailability. These alterations have the potential to adversely affect patient exposure and subsequent therapeutic outcomes. Few studies have been conducted assessing the contribution of MCT isoforms to overall drug disposition and the impact of MCT modulation on drug pharmacokinetics and disposition. The impact of MCT function on drug pharmacokinetics has been most extensively characterized for GHB $(5,76)$. The aim of this section is to summarize work assessing the impact of MCTs on drug disposition specifically focusing on the role of MCTs in the renal clearance of GHB. Current studies on the impact of MCTs on the disposition of additional drugs will also be summarized.

\section{GHB}

GHB is a naturally occurring short-chain fatty acid formed from $\gamma$-aminobutyric acid (GABA) that is found in the mammalian brain, heart, liver and kidney (77). It acts potentially as a neuromodulator through binding to the GABA(B) receptor (78). In addition, GHB is formed from the precursors $\gamma$-butyrolactone and 1,4-butanediol (79). Therapeutically, GHB is approved in the US to treat narcolepsy (marketed as Xyrem ${ }^{\circledR}$ ) (80) and in Europe for the treatment of alcohol withdrawal (81). However, abuse of GHB is widespread; it is used by body builders for its growth hormone releasing properties (82), by drug abusers as a recreational drug for its euphoric effects (83), and in drugfacilitated sexual assault due to its sedative/hypnotic effects (84). The increased abuse of GHB has lead to a rise in associated overdoses and fatalities (82). Adverse events associated with GHB overdose are principally characterized by central nervous system and respiratory depression as well as cardiovascular and gastrointestinal effects with symptoms 
including seizures, dizziness, nausea, vomiting and unconsciousness potentially leading to coma and death. (82) Currently, the treatment of GHB overdose is limited to supportive care; physostigmine and naloxone have been tried as antidotes with minimal success (79).

GHB pharmacokinetics have been demonstrated to be nonlinear in humans $(85-88)$ and rats $(89,90)$, with total clearance decreasing as a function of increasing dose. Several mechanisms contribute to the observed nonlinear pharmacokinetics including capacity-limited metabolism $(85,87,89,90)$, saturable absorption (91), and nonlinear renal clearance (6). While metabolic clearance represents the predominant elimination pathway for GHB (77), renal clearance becomes increasingly important in overdose situations with high urinary concentrations reported in humans $(92,93)$. In contrast to the observed changes in total clearance with increasing dose, renal clearance increases in a dose-dependent manner in rats (6). Furthermore, the fraction of GHB excreted in urine increases tenfold (3\% to $30 \%$ ) over the dose range of 108-208 mg/h per kilogram (6). These dosedependent increases suggest the involvement of active renal reabsorption which is saturated at high concentrations.

In vitro studies have characterized the renal transport mechanisms of GHB and elucidated the MCT isoforms contributing to GHB reuptake. Studies were conducted in rat kidney membrane vesicles, a human kidney cell line (HK2 cells) and rat MCT1 transfected MDA-MB231 cells. Studies conducted in rat brush border (BBM) and basolateral (BLM) membrane vesicles isolated from rat kidney cortex characterized the renal transport mechanism (12). GHB and L-lactate both undergo $\mathrm{pH}$ - and sodium-dependent uptake in BBM vesicles and $\mathrm{pH}$-dependent uptake in BLM vesicles, suggesting the involvements of proton-dependent and sodiumdependent MCTs (12). MCT1 is expressed at both membranes, although there is greater expression at the BLM; MCT2 is expressed only at the BLM (12). HK-2 cells express MCT1, MCT2 and MCT4 at both the mRNA and protein level, which agrees with expression patterns in the human kidney cortex (62). GHB uptake in HK-2 cells was driven by a pH-gradient, and was inhibited by $\mathrm{CHC}$ suggesting that MCTs, but not SMCTs, were responsible for its uptake in HK2 cells (11). Similar uptake parameters and similar inhibitory effects were observed for GHB and lactate suggesting transport by the same or similar transporters (11). Additionally, GHB uptake was inhibited by $\mathrm{pCMB}$ indicating that MCT2 may not be an important transporter in GHB uptake (11). Silencing RNA for MCT1, 2 and 4 in HK-2 cell studies suggested that GHB is predominantly transported by MCT1 $(10,11)$, among the proton-dependent MCTs. Further studies, conducted in MDA-MB231 cells (endogenous expression of MCT2 and MCT4) and MDA-MB231 cells transfected with rat $\mathrm{MCT} 1$, provided further evidence regarding the specific MCT isoforms involved in GHB renal uptake $(10,12)$. GHB was found to be a substrate for MCT1, 2 and $4(10,11)$. However, based on the expression patterns of MCTs in the kidney, MCT1 is likely the primary isoform responsible for GHB renal uptake.

To further explore the influence of MCT1 on GHB renal reabsorption, studies were conducted to assess the modulation of MCT1-mediated GHB transport through the evaluation of potential inhibitors. Uptake of GHB in MDA-MB231 cells was inhibited by the classic MCT inhibitors $\mathrm{CHC}$, phloretin and pCMB with uptake being approximately $60 \%$ of control cells (10). In rat MCT1-transfected MDA-MB231 cells, GHB uptake was inhibited by phloretin, $\mathrm{CHC}$ and Dlactate (12). GHB uptake was also inhibited to a large extent by L-lactate in rat BBM and BLM vesicles (12). As the inhibition of MCTs results in altered GHB uptake in kidney cells, further studies investigated potential strategies for increasing the renal elimination of GHB by administrating the MCT substrates L-lactate and pyruvate (6). The administration of L-lactate resulted in an approximately twofold increase in renal clearance ( 63 to $118 \mathrm{ml} / \mathrm{h}$ per kilogram) with a concomitant increase in total clearance and decrease in steady-state plasma concentrations (6). These results suggest that administration of MCT inhibitors presents a possible clinical strategy for increasing GHB elimination in overdose cases. Furthermore, administration of L-lactate was well tolerated and with no or minimal changes in blood electrolytes indicating a lack of toxicity (unpublished data). Additional in vitro and in vivo studies were conducted to evaluate novel MCT inhibitors and their potential to increase GHB renal clearance and overall pharmacokinetics. Quercetin, a naturally occurring flavonoid, has been demonstrated to inhibit MCT1 and MCT2-mediated L-lactate uptake (58), therefore, we assessed the inhibitory potential of a range of flavonoid compounds. All flavonoid aglycones that were evaluated inhibited GHB uptake in MCT1-transfected cells, with luteolin, morin, and phloretin resulting in the greatest reduction in $\mathrm{GHB}$ uptake $\left(\mathrm{IC}_{50}\right.$ values of $0.41,6.21$ and $2.57 \mu \mathrm{M}$, respectively) (94). In contrast, the flavonoid glycosides had minimal effects on GHB uptake (94). The high potency of luteolin for inhibiting GHB uptake suggested that this compound may be effective at increasing the renal clearance of GHB. Co-administration with luteolin $(10 \mathrm{mg} /$ $\mathrm{kg}$ ) significantly altered the pharmacokinetic profile of GHB $(1 \mathrm{~g} / \mathrm{kg})$ in rats; renal clearance of GHB increased more than threefold (1.36 to $4.28 \mathrm{ml} / \mathrm{min}$ per kilogram) with a concomitant decrease in the pharmacodynamic effect, return of righting reflex (94). These data suggest that flavonoids such as luteolin are potent MCT inhibitors both in vitro and in vivo.

Additional studies have demonstrated that GHB transport occurs via a carrier-mediated processes in the intestine (91), brain (95), and at the blood-brain barrier (5). MCT expression has been demonstrated along the length of the intestine with MCT1 being the predominant isoform (19). Protein expression of MCT1, MCT2 and MCT4 has been demonstrated in human intestinal Caco-2 cells (unpublished data). Transport studies conducted in Caco-2 cells showed that GHB and D-lactate uptake occurred in a $\mathrm{pH}$ - and proton-dependent manner and similar uptake parameters were observed for GHB and D-lactate which could be inhibited by MCT substrates and inhibitors (unpublished data). These results suggest that GHB's absorption is mediated, at least in part, by MCTs in the human intestine. In situ brain perfusion experiments conducted in rats demonstrated saturable uptake of GHB into various brain regions (5). Furthermore, GHB uptake was inhibited by known MCT inhibitors including lactate and pyruvate, suggesting that GHB is a substrate for MCTs expressed at the blood-brain barrier (5). 


\section{Influence of MCTs on the Disposition of Other Drugs}

While GHB represents the best studied drug substrate of MCTs, a number of other drugs have been demonstrated to be MCT substrates or inhibitors in various in vitro systems. Interestingly, the recent characterization of MCT6 showed that this isoform was involved in the transport of diuretics, such as bumetanide, and did not transport monocarboxylates (3).

Investigations into MCT drug substrates have typically been conducted in tissue specific cell lines and membrane vesicles, including Caco- 2 cells, NBL-2 cells, and MDCK cells as well as in vivo. Caco-2 cells and intestinal membrane vesicles represent effective models to assess transportermediated intestinal absorption. Caco- 2 cells have been demonstrated to express MCT1, 2 and 4 protein (unpublished data from our lab) with MCT1 expression being the predominant form consistent with expression in the human intestine (19). MCT1 is expressed on brush border (apical) membrane of intestinal cells and thereby facilitates the intestinal cell uptake of its substrates. In these systems, MCTs have been demonstrated to transport the $\beta$-lactam antibiotics cefdinir (96) and carindacillin (97,98), salicylic acid (99), pravastatin (100) and atorvastatin (101). Furthermore, the role of MCT1 in the intestinal uptake of the $\beta$-lactam antibiotic prodrug, carindacillin, has been confirmed in rat intestinal brush border membrane vesicles. It is thought that the MCT-mediated uptake of carindacillin contributes to the improved exposure to carbenicillin (97). This concept has also been employed in the design of a gabapentin prodrug, XP13512, which was designed to be a substrate for MCT1, specifically to overcome the poor intestinal absorption of gabapentin. Uptake of XP13512 was demonstrated to be MCT1-mediated in Caco-2, HEK-derived and MDCK cell monolayers (38). In vivo studies on XP13512 demonstrated increased oral absorption and bioavailability in rats and monkeys when compared to gabapentin suggesting that exploitation of MCT-mediated uptake may provide a novel strategy for improving intestinal drug absorption (39).

MCT-mediated uptake of drugs, including statins, probenecid and GHB, has also been demonstrated at the bloodbrain barrier. Tsuji et al. (1993) (102) demonstrated that the uptake of simvastatin acid in bovine brain capillary endothelial cells occurred via a $\mathrm{pH}$-dependent carrier-mediated process. Furthermore, the authors showed that pravastatin had a lower affinity for the same transport process and in addition was able to inhibit the uptake of simvastatin acid (102). Statins were recently identified to be inhibitors of MCT4 with the lipophilic statins (fluvastatin, atorvastatin, lovastatin acid, simvastatin acid and cerivastatin) demonstrating the greatest inhibitory potency (65). Further studies need to be conducted to determine the influence of brain MCT expression on the overall disposition of MCT substrates and the potential therapeutic implications.

\section{CONCLUSIONS}

MCTs represent an important family of transport proteins involved in the transport of endogenous and exogenous compounds. Tissue localization of MCT expression suggests the potential for a large impact on the oral absorption, brain uptake and renal clearance of MCT substrates. The inhibition of the transport of GHB by MCT1 provides a novel strategy for GHB detoxification through increasing renal and total clearance. Further investigations are required to more clearly understand the role of MCTs in drug disposition for a wider range of drug substrates.

\section{ACKNOWLEDGEMENTS}

Support was provided by NIH grants DA14988 and DA023223 and a grant from the Western New York Kidney Foundation/Upstate NY Transplantation Service. MAF received a graduate fellowship from Pfizer Global Research Inc.

\section{REFERENCES}

1. V. N. Jackson, and A. P. Halestrap. The kinetics, substrate, and inhibitor specificity of the monocarboxylate (lactate) transporter of rat liver cells determined using the fluorescent intracellular $\mathrm{pH}$ indicator, 2',7'-bis(carboxyethyl)-5(6)-carboxyfluorescein. $J$. Biol. Chem. 271(2):861-868 (1996), Jan 12.

2. A. P. Halestrap, and N. T. Price. The proton-linked monocarboxylate transporter (MCT) family: structure, function and regulation. Biochem. J. 343(Pt 2):281-299 (1999), Oct 15.

3. Y. Murakami, N. Kohyama, Y. Kobayashi, et al. Functional characterization of human monocarboxylate transporter 6 (SLC16A5). Drug Metab. Dispos. 33(12):1845-1851 (2005), Dec.

4. A. P. Halestrap, and D. Meredith. The SLC16 gene family-from monocarboxylate transporters (MCTs) to aromatic amino acid transporters and beyond. Pflugers Arch. 447(5):619-628 (2004), Feb.

5. I. Bhattacharya, and K. M. Boje. GHB (gamma-hydroxybutyrate) carrier-mediated transport across the blood-brain barrier. J. Pharmacol. Exp. Ther. 311(1):92-98 (2004), Oct.

6. M. E. Morris, K. Hu, and Q. Wang. Renal clearance of gammahydroxybutyric acid in rats: increasing renal elimination as a detoxification strategy. J. Pharmacol. Exp. Ther. 313(3):11941202 (2005), Jun.

7. E. Gopal, Y. J. Fei, M. Sugawara, et al. Expression of slc5a8 in kidney and its role in $\mathrm{Na}(+)$-coupled transport of lactate. $J$. Biol. Chem. 279(43):44522-44532 (2004), Oct 22.

8. M. J. Coady, M. H. Chang, F. M. Charron, et al. The human tumour suppressor gene SLC5A8 expresses a Na+-monocarboxylate cotransporter. J. Physiol. 557(Pt 3):719-731 (2004), Jun 15.

9. S. R. Srinivas, E. Gopal, and L. Zhuang, et al. Cloning and functional identification of slc5a12 as a sodium-coupled lowaffinity transporter for monocarboxylates (SMCT2). Biochem. J. 392(Pt 3):655-664 (2005), Dec 15.

10. Q. Wang, and M. E. Morris. The role of monocarboxylate transporter 2 and 4 in the transport of gamma-hydroxybutyric acid in mammalian cells. Drug Metab. Dispos. 35(8):1393-1399 (2007), Aug.

11. Q. Wang, Y. Lu, and M. E. Morris. Monocarboxylate transporter (MCT) mediates the transport of gamma-hydroxybutyrate in human kidney HK-2 cells. Pharm. Res. 24(6):1067-1078 (2007), Jun.

12. Q. Wang, I. M. Darling, and M. E. Morris. Transport of gammahydroxybutyrate in rat kidney membrane vesicles: Role of monocarboxylate transporters. J. Pharmacol. Exp. Ther. 318 (2):751-761 (2006), Aug.

13. R. C. Poole, and A. P. Halestrap. Transport of lactate and other monocarboxylates across mammalian plasma membranes. Am. J. Physiol. 264(4 Pt 1):C761-782 (1993), Apr.

14. B. Deuticke. Monocarboxylate transport in erythrocytes. $J$. Membr. Biol. 70(2):89-103 (1982).

15. C. M. Kim, J. L. Goldstein, and M. S. Brown. cDNA cloning of $\mathrm{MEV}$, a mutant protein that facilitates cellular uptake of 
mevalonate, and identification of the point mutation responsible for its gain of function. J. Biol. Chem. 267(32):23113-23121 (1992), Nov 15.

16. C. K. Garcia, J. L. Goldstein, R. K. Pathak, R. G. Anderson, and M. S. Brown. Molecular characterization of a membrane transporter for lactate, pyruvate, and other monocarboxylates: implications for the Cori cycle. Cell. 76(5):865-873 (1994), Mar 11.

17. C. Juel, and A. P. Halestrap. Lactate transport in skeletal musclerole and regulation of the monocarboxylate transporter. J. Physiol. 517(Pt 3):633-642 (1999), Jun 15.

18. K. Pierre, and L. Pellerin. Monocarboxylate transporters in the central nervous system: distribution, regulation and function. $J$. Neurochem. 94(1):1-14 (2005), Jul.

19. R. K. Gill, S. Saksena, W. A. Alrefai, et al. Expression and membrane localization of MCT isoforms along the length of the human intestine. Am. J. Physiol. Cell Physiol. 289(4):C846C852 (2005), Oct.

20. G. Chidlow, J. P. Wood, M. Graham, and N. N. Osborne. Expression of monocarboxylate transporters in rat ocular tissues. Am. J. Physiol. Cell Physiol. 288(2):C416-428 (2005), Feb.

21. N. J. Philp, D. Wang, H. Yoon, and L. M. Hjelmeland. Polarized expression of monocarboxylate transporters in human retinal pigment epithelium and ARPE-19 cells. Invest. Ophthalmol. Vis. Sci. 44(4):1716-1721 (2003), Apr.

22. L. Bergersen, E. Johannsson, M. L. Veruki, et al. Cellular and subcellular expression of monocarboxylate transporters in the pigment epithelium and retina of the rat. Neuroscience. 90 (1):319-331 (1999), Apr.

23. O. Chiry, L. Pellerin, F. Monnet-Tschudi, et al. Expression of the monocarboxylate transporter MCT1 in the adult human brain cortex. Brain Res. 1070(1):65-70 (2006), Jan 27.

24. T. Hashimoto, S. Masuda, S. Taguchi, and G. A. Brooks. Immunohistochemical analysis of MCT1, MCT2 and MCT4 expression in rat plantaris muscle. J. Physiol. 567(Pt 1):121-129 (2005), Aug 15.

25. A. Bonen. The expression of lactate transporters (MCT1 and MCT4) in heart and muscle. Eur. J. Appl. Physiol. 86(1):6-11 (2001), Nov

26. M. A. Cuff, and S. P. Shirazi-Beechey. The human monocarboxylate transporter, MCT1: genomic organization and promoter analysis. Biochem. Biophys. Res. Commun. 292 (4):1048-1056 (2002), Apr 12.

27. T. Enoki, Y. Yoshida, J. Lally, H. Hatta, and A. Bonen. Testosterone increases lactate transport, monocarboxylate transporter (MCT) 1 and MCT4 in rat skeletal muscle. $J$. Physiol. 577(Pt 1):433-443 (2006), Nov 15.

28. J. Chenal, and L. Pellerin. Noradrenaline enhances the expression of the neuronal monocarboxylate transporter MCT2 by translational activation via stimulation of PI3K/ Akt and the mTOR/S6K pathway. J. Neurochem. 102(2):389397 (2007), Jul.

29. M. C. Wilson, D. Meredith, J. E. Fox, C. Manoharan, A. J. Davies, and A. P. Halestrap. Basigin (CD147) is the target for organomercurial inhibition of monocarboxylate transporter isoforms 1 and 4: the ancillary protein for the insensitive MCT2 is EMBIGIN (gp70). J. Biol. Chem. 280(29):2721327221 (2005), Jul 22.

30. K. Watanabe-Kaneko, T. Sonoda, Y. Miyagi, T. Yamashita, K. Okuda, and S. Kawamoto. The synaptic scaffolding protein Delphilin interacts with monocarboxylate transporter 2. Neuroreport. 18(5):489-493 (2007), Mar 26.

31. V. N. Jackson, N. T. Price, and A. P. Halestrap. cDNA cloning of MCT1, a monocarboxylate transporter from rat skeletal muscle. Biochim. Biophys. Acta. 1238(2):193-196 (1995), Sep 13.

32. L. Carpenter, R. C. Poole, and A. P. Halestrap. Cloning and sequencing of the monocarboxylate transporter from mouse Ehrlich Lettre tumour cell confirms its identity as MCT1 and demonstrates that glycosylation is not required for MCT1 function. Biochim. Biophys. Acta. 1279(2):157-163 (1996), Mar 13.

33. E. M. Koehler-Stec, I. A. Simpson, S. J. Vannucci, K. T. Landschulz, and W. H. Landschulz. Monocarboxylate transporter expression in mouse brain. Am. J. Physiol. 275(3 Pt 1): E516-E524 (1998), Sep.
34. H. Takanaga, I. Tamai, S. Inaba, et al. cDNA cloning and functional characterization of rat intestinal monocarboxylate transporter. Biochem. Biophys. Res. Commun. 217(1):370-377 (1995), Dec 5 .

35. S. Broer, H. P. Schneider, A. Broer, B. Rahman, B. Hamprecht, and J. W. Deitmer. Characterization of the monocarboxylate transporter 1 expressed in Xenopus laevis oocytes by changes in cytosolic pH. Biochem. J. 333(Pt 1):167-174 (1998), Jul 1.

36. A. Borthakur, R. K. Gill, K. Hodges, K. Ramaswamy, G. Hecht, and P. K. Dudeja. Enteropathogenic Escherichia coli inhibits butyrate uptake in Caco-2 cells by altering the apical membrane MCT1 level. Am. J. Physiol. Gastrointest. Liver Physiol. 290(1): G30-G35 (2006), Jan.

37. M. Cuff, J. Dyer, M. Jones, and S. Shirazi-Beechey. The human colonic monocarboxylate transporter Isoform 1: its potential importance to colonic tissue homeostasis. Gastroenterology. 128 (3):676-686 (2005), Mar.

38. K. C. Cundy, R. Branch, T. Chernov-Rogan, et al. XP13512 [(+/ -)-1-([(alpha-isobutanoyloxyethoxy)carbonyl] aminomethyl)-1cyclohexane acetic acid], a novel gabapentin prodrug: I. Design, synthesis, enzymatic conversion to gabapentin, and transport by intestinal solute transporters. J. Pharmacol. Exp. Ther. 311 (1):315-323 (2004), Oct

39. K. C. Cundy, T. Annamalai, L. Bu, et al. XP13512 [(+/-)-1([(alpha-isobutanoyloxyethoxy)carbonyl] aminomethyl)-1-cyclohexane acetic acid], a novel gabapentin prodrug: II. Improved oral bioavailability, dose proportionality, and colonic absorption compared with gabapentin in rats and monkeys. $J$. Pharmacol. Exp. Ther. 311(1):324-333 (2004), Oct.

40. B. E. Enerson, and L. R. Drewes. Molecular features, regulation, and function of monocarboxylate transporters: implications for drug delivery. J. Pharm. Sci. 92(8):15311544 (2003), Aug.

41. J. Fang, Q. J. Quinones, and T. L. Holman, et al. The H+-linked monocarboxylate transporter (MCT1/SLC16A1): a potential therapeutic target for high-risk neuroblastoma. Mol. Pharmacol. 70(6):2108-2115 (2006), Dec.

42. S. M. Gallagher, J. J. Castorino, D. Wang, and N. J. Philp. Monocarboxylate transporter 4 regulates maturation and trafficking of CD147 to the plasma membrane in the metastatic breast cancer cell line MDA-MB-231. Cancer Res. 67(9):41824189 (2007), May 1.

43. M. A. Cuff, D. W. Lambert, and S. P. Shirazi-Beechey. Substrate-induced regulation of the human colonic monocarboxylate transporter, MCT1. J. Physiol. 539(Pt 2):361-371 (2002), Mar 1.

44. T. Hashimoto, R. Hussien, S. Oommen, K. Gohil, and G. A. Brooks. Lactate sensitive transcription factor network in L6 cells: activation of MCT1 and mitochondrial biogenesis. Faseb J. 21(10):2602-2612 (2007), Aug.

45. M. L. Wahl, J. A. Owen, R. Burd, et al. Regulation of intracellular $\mathrm{pH}$ in human melanoma: potential therapeutic implications. Mol. Cancer Ther. 1(8):617-628 (2002), Jun.

46. S. P. Mathupala, C. B. Colen, P. Parajuli, and A. E. Sloan Lactate and malignant tumors: a therapeutic target at the end stage of glycolysis. J. Bioenerg. Biomembr. 39(1):73-77 (2007), Feb.

47. S. P. Mathupala, P. Parajuli, and A. E. Sloan. Silencing of monocarboxylate transporters via small interfering ribonucleic acid inhibits glycolysis and induces cell death in malignant glioma: an in vitro study. Neurosurgery. 55(6):1410-1419 (2004) Dec, discussion 1419.

48. P. Kirk, M. C. Wilson, C. Heddle, M. H. Brown, A. N. Barclay, and A. P. Halestrap. CD147 is tightly associated with lactate transporters MCT1 and MCT4 and facilitates their cell surface expression. Embo J. 19(15):3896-3904 (2000), Aug 1.

49. M. C. Wilson, D. Meredith, and A. P. Halestrap. Fluorescence resonance energy transfer studies on the interaction between the lactate transporter MCT1 and CD147 provide information on the topology and stoichiometry of the complex in situ. $J$. Biol. Chem. 277(5):3666-3672 (2002), Feb 1.

50. C. Manoharan, M. C. Wilson, R. B. Sessions, and A. P. Halestrap. The role of charged residues in the transmembrane helices of monocarboxylate transporter 1 and its ancillary protein basigin in determining plasma membrane expression 
and catalytic activity. Mol. Membr. Biol. 23(6):486-498 (2006), Nov-Dec.

51. C. K. Garcia, M. S. Brown, R. K. Pathak, and J. L. Goldstein. cDNA cloning of MCT2, a second monocarboxylate transporter expressed in different cells than MCT1. J. Biol. Chem. 270 (4):1843-1849 (1995), Jan 27.

52. V. N. Jackson, N. T. Price, L. Carpenter, and A. P. Halestrap. Cloning of the monocarboxylate transporter isoform MCT2 from rat testis provides evidence that expression in tissues is species-specific and may involve post-transcriptional regulation. Biochem. J. 324(Pt 2):447-453 (1997), Jun 1.

53. R. Y. Lin, J. C. Vera, R. S. Chaganti, and D. W. Golde. Human monocarboxylate transporter 2 (MCT2) is a high affinity pyruvate transporter. J. Biol. Chem. 273(44):28959-28965 (1998), Oct 30

54. K. Pierre, L. Pellerin, R. Debernardi, B. M. Riederer, and P. J. Magistretti. Cell-specific localization of monocarboxylate transporters, MCT1 and MCT2, in the adult mouse brain revealed by double immunohistochemical labeling and confocal microscopy. Neuroscience. 100(3):617-627 (2000).

55. L. Pellerin, G. Pellegri, J. L. Martin, and P. J. Magistretti. Expression of monocarboxylate transporter mRNAs in mouse brain: support for a distinct role of lactate as an energy substrate for the neonatal vs. adult brain. Proc. Natl. Acad. Sci. U. S. A. 95(7):3990-3995 (1998), Mar 31.

56. S.X. Zhang, T.R. Searcy, Y. Wu, D. Gozal, and Y. Wang. Alternative promoter usage and alternative splicing contribute to mRNA heterogeneity of mouse monocarboxylate transporter 2. Physiol. Genomics. 32:95-104 (2007), Oct 2.

57. L. Bergersen, O. Waerhaug, J. Helm, et al. A novel postsynaptic density protein: the monocarboxylate transporter MCT2 is colocalized with delta-glutamate receptors in postsynaptic densities of parallel fiber-Purkinje cell synapses. Exp. Brain Res. 136 (4):523-534 (2001), Feb.

58. S. Broer, A. Broer, H. P. Schneider, C. Stegen, A. P. Halestrap, and J. W. Deitmer. Characterization of the high-affinity monocarboxylate transporter MCT2 in Xenopus laevis oocytes. Biochem. J. 341(Pt 3):529-535 (1999), Aug 1.

59. N. J. Philp, H. Yoon, and E. F. Grollman. Monocarboxylate transporter MCT1 is located in the apical membrane and MCT3 in the basal membrane of rat RPE. Am. J. Physiol. 274(6 Pt 2): R1824-R1828 (1998), Jun.

60. E. F. Grollman, N. J. Philp, P. McPhie, R. D. Ward, and B. Sauer. Determination of transport kinetics of chick MCT3 monocarboxylate transporter from retinal pigment epithelium by expression in genetically modified yeast. Biochemistry. 39 (31):9351-9357 (2000), Aug 8.

61. S. Zhu, P. J. Goldschmidt-Clermont, and C. Dong. Inactivation of monocarboxylate transporter MCT3 by DNA methylation in atherosclerosis. Circulation. 112(9):1353-1361 (2005), Aug 30.

62. Q. Wang, Y. Lu, M. Yuan, I. M. Darling, E. A. Repasky, and M. E. Morris. Characterization of monocarboxylate transport in human kidney HK-2 cells. Mol. Pharm. 3(6):675-685 (2006), Nov-Dec.

63. M. C. Wilson, V. N. Jackson, C. Heddle, et al. Lactic acid efflux from white skeletal muscle is catalyzed by the monocarboxylate transporter isoform MCT3. J. Biol. Chem. 273(26):15920-15926 (1998), Jun 26.

64. J. E. Manning Fox, D. Meredith, and A. P. Halestrap. Characterisation of human monocarboxylate transporter 4 substantiates its role in lactic acid efflux from skeletal muscle. J. Physiol. 529(Pt 2):285-293 (2000), Dec 1.

65. M. Kobayashi, Y. Otsuka, S. Itagaki, T. Hirano, and K. Iseki. Inhibitory effects of statins on human monocarboxylate transporter 4. Int. J. Pharm. 317(1):19-25 (2006), Jul 6.

66. N. T. Price, V. N. Jackson, and A. P. Halestrap. Cloning and sequencing of four new mammalian monocarboxylate transporter (MCT) homologues confirms the existence of a transporter family with an ancient past. Biochem. J. 329(Pt 2): 321-328 (1998), Jan 15.

67. K. C. Cundy. Novel uses of drug transporters for drug delivery: a case study with gabapentin. http://www.aapspharmaceutica. com/meetings/files/43/cundy.ppt. Accessed 05 February 2008.
68. W. E. Visser, E. C. Friesema, J. Jansen, and T. J. Visser. Thyroid hormone transport by monocarboxylate transporters. Best Pract. Res. Clin. Endocrinol. Metab. 21(2):223-236 (2007), Jun.

69. T. Ramadan, S. M. Camargo, V. Summa, et al. Basolateral aromatic amino acid transporter TAT1 (Slc16a10) functions as an efflux pathway. J. Cell Physiol. 206(3):771-779 (2006), Mar.

70. D. K. Kim, Y. Kanai, A. Chairoungdua, H. Matsuo, S. H. Cha, and $\mathrm{H}$. Endou. Expression cloning of a $\mathrm{Na}+$-independent aromatic amino acid transporter with structural similarity to $\mathrm{H}$ +/monocarboxylate transporters. J. Biol. Chem. 276(20):1722117228 (2001), May 18.

71. E. C. Friesema, S. Ganguly, A. Abdalla, J. E. Manning Fox, A. P. Halestrap, and T. J. Visser. Identification of monocarboxylate transporter 8 as a specific thyroid hormone transporter. J. Biol. Chem. 278(41):40128-40135 (2003), Oct 10.

72. E. C. Friesema, G. G. Kuiper, J. Jansen, T. J. Visser, and M. H. Kester. Thyroid hormone transport by the human monocarboxylate transporter 8 and its rate-limiting role in intracellular metabolism. Mol. Endocrinol. 20(11):2761-2772 (2006), Nov.

73. C. E. Schwartz, and R. E. Stevenson. The MCT8 thyroid hormone transporter and Allan-Herndon-Dudley syndrome. Best Pract. Res. Clin. Endocrinol. Metab. 21(2):307-321 (2007), Jun.

74. M. A. Chiong, K. G. Sim, K. Carpenter, et al. Transient multiple acyl-CoA dehydrogenation deficiency in a newborn female caused by maternal riboflavin deficiency. Mol. Genet. Metab. 92 (1-2):109-114 (2007), Sep-Oct.

75. T. Hirai, Y. Fukui, and K. Motojima. PPARalpha agonists positively and negatively regulate the expression of several nutrient/drug transporters in mouse small intestine. Biol. Pharm. Bull. 30(11):2185-2190 (2007), Nov.

76. I. Bhattacharya, and K. M. Boje. Potential gamma-hydroxybutyric acid (GHB) drug interactions through blood-brain barrier transport inhibition: a pharmacokinetic simulation-based evaluation. $J$. Pharmacokinet. Pharmacodyn. 33(5):657-681 (2006), Oct.

77. M. Maitre. The gamma-hydroxybutyrate signalling system in brain: organization and functional implications. Prog. Neurobiol. 51(3):337-361 (1997), Feb.

78. O. C. Snead 3rd. Evidence for a G protein-coupled gammahydroxybutyric acid receptor. J. Neurochem. 75(5):1986-1996 (2000), Nov.

79. P. E. Mason, and W. P. Kerns 2nd. Gamma hydroxybutyric acid (GHB) intoxication. Acad. Emerg. Med. 9(7):730-739 (2002), Jul.

80. M. Mamelak, M. B. Scharf, and M. Woods. Treatment of narcolepsy with gamma-hydroxybutyrate. A review of clinical and sleep laboratory findings. Sleep. 9(1 Pt 2):285-289 (1986).

81. L. Gallimberti, M. R. Spella, C. A. Soncini, and G. L. Gessa. Gamma-hydroxybutyric acid in the treatment of alcohol and heroin dependence. Alcohol. 20(3):257-262 (2000), Apr.

82. M. S. Okun, L. A. Boothby, R. B. Bartfield, and P. L. Doering. GHB: an important pharmacologic and clinical update. $J$. Pharm. Pharm. Sci. 4(2):167-175 (2001), Aug.

83. C. G. Wong, K. M. Gibson, and O. C. Snead 3rd. From the street to the brain: neurobiology of the recreational drug gamma-hydroxybutyric acid. Trends Pharmacol. Sci. 25(1):2934 (2004), Jan.

84. R. H. Schwartz, R. Milteer, and M. A. LeBeau. Drug-facilitated sexual assault ('date rape'). South Med J. 93(6):558-561 (2000), Jun.

85. P. Palatini, L. Tedeschi, and G. Frison, et al. Dose-dependent absorption and elimination of gamma-hydroxybutyric acid in healthy volunteers. Eur. J. Clin. Pharmacol. 45(4):353-356 (1993).

86. M. B. Scharf, A. A. Lai, B. Branigan, R. Stover, and D. B. Berkowitz. Pharmacokinetics of gamma hydroxybutyrate (GHB) in narcoleptic patients. Sleep. 21(5):507-514 (1998), Aug 1.

87. S. D. Ferrara, S. Zotti, L. Tedeschi, et al. Pharmacokinetics of gamma-hydroxybutyric acid in alcohol dependent patients after single and repeated oral doses. Br. J. Clin. Pharmacol. 34 (3):231-235 (1992), Sep.

88. S. D. Ferrara, L. Tedeschi, G. Frison, et al. Effect of moderate or severe liver dysfunction on the pharmacokinetics of gamma- 
hydroxybutyric acid. Eur. J. Clin. Pharmacol. 50(4):305-310 (1996).

89. J. T Lettieri, and H. L. Fung. Dose-dependent pharmacokinetics and hypnotic effects of sodium gamma-hydroxybutyrate in the rat. J. Pharmacol. Exp. Ther. 208(1):7-11 (1979), Jan.

90. J. Lettieri, and H. L. Fung. Improved pharmacological activity via pro-drug modification: comparative pharmacokinetics of sodium gamma-hydroxybutyrate and gamma-butyrolactone. Res. Commun. Chem. Pathol. Pharmacol. 22(1):107-118 (1978), Oct.

91. C. Arena, and H. L. Fung. Absorption of sodium gammahydroxybutyrate and its prodrug gamma-butyrolactone: relationship between in vitro transport and in vivo absorption. $J$. Pharm. Sci. 69(3):356-358 (1980), Mar.

92. K. A. Sporer, R. L. Chin, J. E. Dyer, and R. Lamb. Gammahydroxybutyrate serum levels and clinical syndrome after severe overdose. Ann. Emerg. Med. 42(1):3-8 (2003), Jul.

93. D. L. Zvosec, S. W. Smith, J. R. McCutcheon, J. Spillane, B. J. Hall, and E. A. Peacock. Adverse events, including death, associated with the use of 1,4-butanediol. N. Engl. J. Med. 344 (2):87-94 (2001), Jan 11.

94. Q. Wang, and M. E. Morris. Flavonoids modulate monocarboxylate transporter-1-mediated transport of gamma-hydroxybutyrate in vitro and in vivo. Drug Metab. Dispos. 35(2):201-208 (2007), Feb.

95. J. Benavides, J. F. Rumigny, J. J. Bourguignon, C. G. Wermuth, P. Mandel, and M. Maitre. A high-affinity, Na+-dependent uptake system for gamma-hydroxybutyrate in membrane vesicles prepared from rat brain. J. Neurochem. 38(6):15701575 (1982), Jun.

96. A. Tsuji, I. Tamai, M. Nakanishi, T. Terasaki, and S. Hamano. Intestinal brush-border transport of the oral cephalosporin antibiotic, cefdinir, mediated by dipeptide and monocarboxylic acid transport systems in rabbits. J. Pharm. Pharmacol. 45 (11):996-998 (1993), Nov.
97. Y. H. Li, K. Ito, Y. Tsuda, R. Kohda, H. Yamada, and T. Itoh. Mechanism of intestinal absorption of an orally active betalactam prodrug: uptake and transport of carindacillin in Caco-2 cells. J. Pharmacol. Exp. Ther. 290(3):958-964 (1999), Sep.

98. Y. H. Li, M. Tanno, T. Itoh, and H. Yamada. Role of the monocarboxylic acid transport system in the intestinal absorption of an orally active beta-lactam prodrug: carindacillin as a model. Int. J. Pharm. 191(2):151-159 (1999), Nov 30.

99. S. Neuhoff, A. L. Ungell, I. Zamora, and P. Artursson. pHdependent passive and active transport of acidic drugs across Caco-2 cell monolayers. Eur. J. Pharm. Sci. 25(2-3):211-220 (2005), Jun.

100. I. Tamai, H. Takanaga, H. Maeda, T. Ogihara, M. Yoneda, and A. Tsuji. Proton-cotransport of pravastatin across intestinal brushborder membrane. Pharm. Res. 12(11):1727-1732 (1995), Nov.

101. X. Wu, L. R. Whitfield, and B. H. Stewart. Atorvastatin transport in the Caco-2 cell model: contributions of P-glycoprotein and the proton-monocarboxylic acid co-transporter. Pharm. Res. 17(2):209-215 (2000), Feb.

102. A. Tsuji, A. Saheki, I. Tamai, and T. Terasaki. Transport mechanism of 3-hydroxy-3-methylglutaryl coenzyme A reductase inhibitors at the blood-brain barrier. J. Pharmacol. Exp. Ther. 267(3):1085-1090 (1993), Dec.

103. P. Settle, K. Mynett, P. Speake, et al. Polarized lactate transporter activity and expression in the syncytiotrophoblast of the term human placenta. Placenta. 25(6):496-504 (2004), Jul.

104. K. S. Dimmer, B. Friedrich, F. Lang, J. W. Deitmer, and S. Broer. The low-affinity monocarboxylate transporter MCT4 is adapted to the export of lactate in highly glycolytic cells. Biochem. J. 350(Pt 1):219-227 (2000), Aug 15.

105. S. Y. Chan, J. A. Franklyn, H. N. Pemberton, et al. Monocarboxylate transporter 8 expression in the human placenta: the effects of severe intrauterine growth restriction. J. Endocrinol. 189(3):465-471 (2006), Jun. 